\title{
Can We Measure the Learning Curve of Colonoscopy Using Polyp Detection Rate?
}

\author{
Jin Young Yoon and Jae Myung Cha \\ Division of Gastroenterology, Department of Internal Medicine, Kyung Hee University Hospital at Gangdong, Kyung Hee University School of \\ Medicine, Seoul, Korea
}

See “The Colonoscopist's Expertise Affects The Characteristics of Detected Polyps” by Da Kyoung Jung, Tae Oh Kim, Mi Seon Kang, et al., on page 61-68.

Competence in colonoscopy procedures is a critical component for gastroenterology fellowship training programs. However, not only specific measures of colonoscopy competence but also a precisely structured curriculum is lacking. The approach to determine whether a trainee has achieved competence in colonoscopy procedures remains debatable. With respect to technical competence, successful learning has been defined as the ability to intubate the cecum sufficiently. ${ }^{1}$ Therefore, a common approach has been to establish a threshold number of procedures required to achieve a cecal intubation rate of $\geq 90 \%$. $^{2}$ On the other hand, the adenoma detection rate (ADR) may be a reflection of clinical competence. ${ }^{3}$ As we know, $\mathrm{ADR}$ is considered as the primary measure of the quality of mucosal inspection and the single most important quality measure in colonoscopy. ${ }^{1}$ One issue regarding ADR is that many practices find it difficult to determine the ADR because it requires a combination of endoscopic and histologic findings. Therefore, as a surrogate for ADR, many studies ${ }^{4-7}$ have suggested polyp detection rate (PDR), which is defined as the number of patients with more than one polyp removed during screening colonoscopy. PDR has the advantage of not

Received: December 19, 2015 Accepted: December 27, 2015

Correspondence: Jae Myung Cha

Department of Internal Medicine, Kyung Hee University Hospital at Gangdong, Kyung Hee University School of Medicine, 892 Dongnam-ro, Gangdong-gu, Seoul 05278, Korea

Tel: +82-2-440-6113, Fax: +82-2-440-6296, E-mail: drcha@khu.ac.kr

cc This is an Open Access article distributed under the terms of the Creative Commons Attribution Non-Commercial License (http://creativecommons.org/ licenses/by-nc/3.0) which permits unrestricted non-commercial use, distribution, and reproduction in any medium, provided the original work is properly cited. requiring manual entry of pathological data and has been reported to correlate well with ADR in previous studies. ${ }^{4-7}$ However, no studies investigating whether PDR remains an accurate correlate to ADR when used prospectively in quality improvement programs exist. Furthermore, PDR has the risk of unwarranted polypectomy as the colonoscopist may remove non-neoplastic polyps, which are not considered to have a risk of becoming cancer, such as diminutive hyperplastic polyps at the sigmoid colon or rectum. Therefore, PDR is not endorsed as a quality indicator to be used independently of ADR until now. However, considering the ease of application of PDR, further studies of its use are desirable and considered necessary to establish its appropriateness.

In this issue of Clinical Endoscopy, Jung et al. ${ }^{8}$ discriminatively analyzed data on PDR as a surrogate marker used for determining learning curves. Some studies in the past have investigated the detection rates of apprentices. On comparing initial and final ADR values of trainees, no significant differences were verified. ${ }^{3,9,10}$ In accordance with previous studies, no significant difference between ADR values after 50 and 200 consecutive colonoscopies was reported. Jung et al. ${ }^{8}$ suggest that the quality of a colonoscopy, measured using the PDR, may improve when performed by experienced fellows. Of interest, PDR was calculated based on the percentage of patients who had at least one polyp (method A), which was commonly used in previous studies, ${ }^{4-7}$ and according to the percentage of detected lesions (method B). As it is impossible to distinguish between two subjects with different numbers of polyps using method A, method B was also used in this study 
to overcome the limitation of method A. There was no proportional correlation between the PDR and increasing experience in colonoscopy using method A; however, with method $B$, the PDR increased after 400 colonoscopies $(p=0.0209$ ). Using method B, the detection rates of small polyps $(<5 \mathrm{~mm}$; $p=0.0015)$ and polyps in proximal sites $(p=0.0050)$ increased after 300 colonoscopies. The study showed that PDR increased with increasing experience of the fellow, and with the experience of more than 300 colonoscopies, small, clinically insignificant polyps and polyps deemed difficult to detect could be detected at higher rates. ${ }^{8}$ These findings may suggest that the colonoscopist's expertise is an important factor in the quality of screening colonoscopy.

Among healthy asymptomatic patients undergoing screening colonoscopy, adenomas should be detected in $25 \%$ of men and $15 \%$ of women over the age of 50 years. ${ }^{1}$ Personal ADR values ranged between $16 \%$ and $24 \%$ in beginners experienced below 100 colonoscopic cases in this study. Although it was identified that $\mathrm{ADR}$ values are capable of reflecting trainee performance, trainees enrolled in this study fulfilled quality standards from the very beginning. Thus, early ADR values might represent merged parameters, including the trainer's observation. We notice that the nature of training has to be kept in mind when interpreting these results.

This study's findings should be interpreted cautiously. There is the possibility of selection bias, as this study was a retrospective, single-center study based on only three fellows. Authors included only asymptomatic patients older than 50 years who underwent screening colonoscopy; however, PDR may be influenced by previous screening examination of the colon and rectum. In this study, only colonoscopies for which the withdrawal time (WT) exceeded 6 minutes were assessed; however, whether a WT of more than 6 minutes is optimal performance target for fellows remains unclear. Despite these limitations, this study was the first one on this issue suggesting that the colonoscopist's expertise affects the characteristics of detected polyps, in addition to technological advances and improvements in the field of view and imaging. Therefore, based on the current study by Jung et al., ${ }^{8}$ multicenter, randomized, prospective studies are warranted on this issue.

Conflicts of Interest

The authors have no financial conflicts of interest.

\section{REFERENCES}

1. Rex DK, Schoenfeld PS, Cohen J, et al. Quality indicators for colonoscopy. Am J Gastroenterol 2015;110:72-90.

2. Ward ST, Mohammed MA, Walt R, Valori R, Ismail T, Dunckley P. An analysis of the learning curve to achieve competency at colonoscopy using the JETS database. Gut 2014;63:1746-1754.

3. Lee SH, Chung IK, Kim SJ, et al. An adequate level of training for technical competence in screening and diagnostic colonoscopy: a prospective multicenter evaluation of the learning curve. Gastrointest Endosc 2008;67:683-689.

4. Williams JE, Le TD, Faigel DO. Polypectomy rate as a quality measure for colonoscopy. Gastrointest Endosc 2011;73:498-506.

5. Francis DL, Rodriguez-Correa DT, Buchner A, Harewood GC, Wallace $\mathrm{M}$. Application of a conversion factor to estimate the adenoma detection rate from the polyp detection rate. Gastrointest Endosc 2011;73:493-497.

6. Williams JE, Holub JL, Faigel DO. Polypectomy rate is a valid quality measure for colonoscopy: results from a national endoscopy database. Gastrointest Endosc 2012;75:576-582.

7. Gohel TD, Burke CA, Lankaala P, et al. Polypectomy rate: a surrogate for adenoma detection rate varies by colon segment, gender, and endoscopist. Clin Gastroenterol Hepatol 2014;12:1137-1142.

8. Jung DK, Kim TO, Kang MS, Kim MS, Kim MS, Moon YS. The colonoscopist's expertise affects the characteristics of detected polyps. Clin Endosc 2016;49;61-68.

9. Spier BJ, Benson M, Pfau PR, Nelligan G, Lucey MR, Gaumnitz EA. Colonoscopy training in gastroenterology fellowships: determining competence. Gastrointest Endosc 2010;71:319-324.

10. Gromski MA, Miller CA, Lee SH, et al. Trainees' adenoma detection rate is higher if $\geq 10$ minutes is spent on withdrawal during colonoscopy. Surg Endosc 2012;26:1337-1342. 